Full length article

\title{
Health insurance, alcohol and tobacco use among pregnant and non-pregnant women of reproductive age
}

\author{
Qiana L. Brown ${ }^{a}$, Deborah S. Hasin ${ }^{a, b}, *$, Katherine M. Keyes ${ }^{a}$, David S. Fink $^{a}$, \\ Orson Ravenell ${ }^{\mathrm{c}}$, Silvia S. Martins ${ }^{\mathrm{a}}$
}

a Department of Epidemiology, Mailman School of Public Health, Columbia University, New York, NY, USA

${ }^{\mathrm{b}}$ New York State Psychiatric Institute, New York, NY, USA

' Kraemer Women's Care, Columbia, SC, USA

\section{A R T I C L E I N F O}

\section{Article history:}

Received 14 December 2015

Received in revised form 22 June 2016

Accepted 2 July 2016

Available online 12 July 2016

\section{Keywords:}

Tobacco

Alcohol

Pregnancy

Health insurance

\begin{abstract}
A B S T R A C T
Background: Understanding the relationship between health insurance coverage and tobacco and alcohol use among reproductive age women can provide important insight into the role of access to care in preventing tobacco and alcohol use among pregnant women and women planning to become pregnant. Methods: We examined the association between health insurance coverage and both past month alcohol use and past month tobacco use in a nationally representative sample of women age 12-44 years old, by pregnancy status. The women $(n=97,788)$ were participants in the National Survey of Drug Use and Health (NSDUH) in 2010-2013. Logistic regression models assessed the association between health insurance (insured versus uninsured), past month tobacco and alcohol use, and whether this was modified by pregnancy status.

Results: Pregnancy status significantly moderated the relationship between health insurance and tobacco use ( $p$-value $\leq 0.01$ ) and alcohol use ( $p$-value $\leq 0.01)$. Among pregnant women, being insured was associated with lower odds of alcohol use (adjusted odds ratio [AOR] $=0.47 ; 95 \%$ confidence interval $[C I]=0.27-0.82)$, but not associated with tobacco use ( $A O R=1.14 ; 95 \% C I=0.73-1.76)$. Among nonpregnant women, being insured was associated with lower odds of tobacco use (AOR=0.67; 95\% $\mathrm{CI}=0.63-0.72)$, but higher odds of alcohol use $(\mathrm{AOR}=1.23 ; 95 \% \mathrm{CI}=1.15-1.32)$.

Conclusion: Access to health care, via health insurance coverage is a promising method to help reduce alcohol use during pregnancy. However, despite health insurance coverage, tobacco use persists during pregnancy, suggesting missed opportunities for prevention during prenatal visits.
\end{abstract}

(c) 2016 Elsevier Ireland Ltd. All rights reserved.

\section{Background}

Tobacco and alcohol are the most commonly used substances among women of childbearing age including those who are pregnant (Floyd et al., 2008). During pregnancy, both alcohol use (Hankin, 2002; Li et al., 2012; Maier and West, 2001; Terplan et al., 2014) and tobacco use (Coleman-Cowger et al., 2014; Holtrop et al., 2010; Mund et al., 2013) are leading causes of preventable adverse health outcomes for mothers and babies. Of the most severe (and preventable) health outcomes associated with prenatal alcohol exposure is fetal alcohol syndrome (FAS; Hankin, 2002; Maier

\footnotetext{
* Correspondence to: Department of Psychiatry, Columbia University Medical Center, 1051 Riverside Drive \#123, New York, NY 10032, USA.

E-mail addresses: deborah.hasin@gmail.com,dsh2@cumc.columbia.edu (D.S. Hasin).
}

and West, 2001). In addition, prenatal alcohol use is associated with a host of other poor health outcomes including, spontaneous abortion, neurodevelopment problems, and pre- and post-natal growth deficits (Floyd et al., 2008). Similarly, prenatal tobacco use is associated with pre-term and low birth-weight deliveries and infant mortality (Dietz et al., 2010). During pregnancy, there is no safe level of alcohol consumption (Floyd et al., 2008), and regardless of pregnancy status, there is no safe level of exposure to smoke from tobacco products (US Department of Health and Human Services, 2010). Therefore, identifying factors that may reduce the risk of tobacco and alcohol use among reproductive age women, especially those who are already pregnant, is crucial to promoting the health of mother and child. The access to health care provided by insurance may be an important factor to consider in efforts to reduce alcohol and tobacco use among this population.

Health insurance is an important element in one's access to health care (Chen et al., 2015; Newacheck et al., 1998; Zuvekas and 
Taliaferro, 2003). Insurance coverage and health care access among women of reproductive age could improve maternal and child health (D'Angelo et al., 2015) by improving access to timely and adequate prenatal care (D'Angelo et al., 2007; Egerter et al., 2002; Jarvis et al., 2011). For example, an estimated $30 \%$ of women receive preconception provider-delivered health counseling on preparing for a healthy pregnancy and baby, and approximately $90 \%$ of women receive health check-ups postpartum and between pregnancies (D'Angelo et al., 2007). Furthermore, pregnant women may be more motivated to stop using tobacco and alcohol than nonpregnant women (Ethen et al., 2009; Floyd et al., 2008; Havens et al., 2009; Terplan et al., 2012; Tong et al., 2009), and access to health care through health insurance may help facilitate this process by increasing exposure to prenatal visits, which present opportunities for providers to engage patients in alcohol and tobacco use prevention interventions. Therefore, health insurance coverage may be indirectly associated with reductions in tobacco and alcohol use via increased access to prenatal care where substance use prevention can occur. However, investigating a potential direct effect between health insurance coverage and tobacco and alcohol use among reproductive age women is an essential first step in helping to determine if there is an effect to be mediated. Furthermore, given that substance use disorder treatment, as well as maternal and child health care are part of the Essential Health Benefits covered by the Affordable Care Act (Beronio et al., 2013; Department of the Treasury et al., 2013; Office of Legislative Counsel, 2010), this may encourage both patients and providers to engage in discussions around alcohol and tobacco use prevention during prenatal visits.

Several studies that examined tobacco and alcohol use during pregnancy did not incorporate information on insurance status (Ethen et al., 2009; Havens et al., 2009; Kratz and Vaughan, 2012; Muhuri and Gfroerer, 2009). In a study that did explore health insurance and substance use in pregnant and non-pregnant women of reproductive age (Terplan et al., 2012), alcohol and illicit drug use were pooled, and tobacco was not addressed. In another study, the prevalence of tobacco use by insurance status was reported, pooling use during, before, and after pregnancy (Tong et al., 2009). However, this study did not adjust for potential confounding. Furthermore, clinical implications and health risk of use during pregnancy differs compared to use before or after pregnancy. Other studies have focused specifically on low-income pregnant women or emphasized the role of Medicaid in tobacco use during pregnancy (Adams et al., 2013; Jarlenski et al., 2014; Ma et al., 2005). Thus, little is known about the role that health insurance plays in tobacco and alcohol use among pregnant and non-pregnant women of reproductive age. Given the widespread availability of health insurance coverage in the United States through the Affordable Care Act and the substance use prevention services covered (Beronio et al., 2013; Department of the Treasury et al., 2013; Office of Legislative Counsel, 2010), understanding the relationship of health insurance to tobacco and alcohol use among pregnant women and reproductive age women who may become pregnant can have important implications for maternal and child health. Specifically, insurance coverage may serve as a universal prevention intervention to reduce tobacco and alcohol use among pregnant women and women contemplating pregnancy. Therefore, we conducted a study to fill this gap in knowledge. In this study, we investigated the relationship between health insurance and both past month tobacco use and past month alcohol use among a nationally representative sample of reproductive age women in the United States, controlling for important covariates (e.g., age, race/ethnicity, education, marital status, poverty). We examined whether the relationship between health insurance and alcohol or tobacco use differed between pregnant and non-pregnant women. We hypothesized that pregnancy status would moderate the relationship between health insurance status and tobacco and alcohol use, such that having health insurance would be associated with lower odds of tobacco and alcohol use among both pregnant and non-pregnant women, and that this inverse relationship would be stronger among pregnant women, given that pregnant women may be more motivated to quit substance use than non-pregnant women (Ethen et al., 2009; Floyd et al., 2008; Havens et al., 2009; Terplan et al., 2012; Tong et al., 2009).

\section{Methods}

\subsection{Data source}

We used data from the National Survey of Drug Use and Health (NSDUH) public use files (Substance Abuse and Mental Health Services Administration (SAMHSA), 2012). The NSDUH annual surveys assess substance use and other behaviors in nationally representative samples of the non-institutionalized U.S. citizen population aged 12 years and older. Each year, participants are selected via independent multistage area probability samples in each of the 50 States and the District of Columbia. Trained interviewers explained all study procedures to respondents and obtained informed consent before starting the interviews. Surveys were administered using computer-assisted personal interviewing (CAPI) and audio computer-assisted self-interviewing (ACASI). The combined use of CAPI and ACASI increases the validity of self-reported data by providing a confidential means for the interviewees to respond to sensitive questions about embarrassing or illegal behaviors (Biondo and Chilcoat, 2014; SAMHSA, 2012). All procedures were approved by the Institutional Review Boards at the Research Triangle Institute (RTI; SAMHSA, 2010, 2011, 2012, 2013a).

For the present analysis, women of reproductive age (12-44 years) were sampled from the NSDUH surveys conducted in 2010 (pregnant $n=899$; not pregnant $n=23,988$; pregnancy status unknown $n=129$ ), 2011 (pregnant $n=830$; not pregnant $n=24,027$; pregnancy status unknown $n=115$ ), 2012 (pregnant $n=776$; not pregnant $n=22,938$; pregnancy status unknown $n=122$ ) and 2013 (pregnant $n=762$; not pregnant $n=23,102$; pregnancy status unknown $n=100$ ). Pooling these participants yielded a final sample of 94,055 non-pregnant women, 3267 pregnant women, and 466 women of unknown pregnancy status for a total sample of 97,788 women. The age range 12-44 years old was selected because the NSDUH only assesses pregnancy status among women in this age group. Survey years 2010-2013 were used because they were the latest waves available at the time of the analyses, and 2010 marks a significant policy shift regarding health insurance availability with the passing of the Affordable Care Act (Department of the Treasury et al., 2013; Office of Legislative Counsel, 2010).

\subsection{Measures}

2.2.1. Outcomes. Two binary outcomes were analyzed - past month tobacco use and past month alcohol use. Past month tobacco use included cigarettes, cigars, pipes, or smokeless tobacco use approximately $84 \%$ of those who reported past month tobacco use reported cigarette use only (i.e., not cigars, pipes, or smokeless tobacco). Respondents endorsing any of these types of tobacco use in the past 30 days were classified as past month tobacco users. Past month alcohol use included any alcohol use. Respondents endorsing use during the past 30 days were classified as past month drinkers.

2.2.2. Exposure. Current health insurance status was the exposure of interest. A binary variable was created to indicate whether participants reported being covered by any health insurance (private 
insurance, Medicaid/CHIP, Medicare, Champus, ChampusVA, VA, Military, or other insurance) or were uninsured.

2.2.3. Moderator. Pregnancy status was the hypothesized moderator of the relationship between health insurance and tobacco and alcohol use. Women, age 12-44 years old were asked about their pregnancy status (i.e., "are you pregnant?"). They could either respond "yes," "no," "don't know," "refused to answer," or leave the answer choice blank. There were a total of 466 who answered "don't know," "refused to answer," or left the answer choice blank. These 466 women and the non-pregnant women (i.e., those who answered "no"; $n=94,055$ ) were combined into the reference category for these analyses.

2.2.4. Covariates. Covariates were chosen based on theoretical relevance supported by prior literature (D’Angelo et al., 2012; Kratz and Vaughan, 2012; Ma et al., 2005; Terplan et al., 2012). Analyses were adjusted for age (12-17 years, $18-25$ years, and 26-44 years); race and ethnicity (non-Hispanic Black/African American, nonHispanic White, Hispanic, other); education (high school diploma or above versus less); marital status (married versus unmarried [i.e., never married, widowed, divorced or separated]); poverty (based on the US Census Bureau's poverty thresholds, which accounts for age, family size, number of children in the household, and family income; SAMHSA, 2013b) - less than $100 \%$ of poverty threshold, $100-199 \%$ of threshold, $200 \%$ or greater than the poverty threshold (reference group); and survey year. In addition, past month alcohol use was included as a covariate when modeling past month tobacco use as the outcome; and past month tobacco use was included as a covariate when modeling past month alcohol use as the outcome.

2.2.5. Descriptive data. Alcohol and tobacco use among pregnant women, by insurance status and trimester was assessed. Differences in alcohol and tobacco use by insurance status within trimesters were tested with design-based F-tests. Additionally, for descriptive purposes, the proportion of reproductive age women who met criteria for past month nicotine dependence according to the Nicotine Dependence Syndrome Scale or the Fagerstrom Test of Nicotine Dependence; and who met the Diagnostic and Statistical Manual of Mental Disorders, Fourth Edition (DSM-IV) criteria for past year alcohol abuse or dependence was examined by pregnancy and insurance status.

\subsection{Statistical analysis}

Descriptive statistics were calculated to examine demographic characteristics by pregnancy and insurance status. In pooled analyses that combined pregnant and non-pregnant women, logistic regression models were used to generate unadjusted and adjusted odds ratios (AOR) and 95\% confidence intervals (CI) to assess the relationship between insurance status and past month tobacco and alcohol use. Effect modification of the relationship between health insurance and alcohol and tobacco use by pregnancy status was assessed by creating multiplicative interaction terms between pregnancy status and insurance status. Each effect modification model included the interaction term, the main effects, and covariates. Based on the statistically significant interaction terms that we found, tobacco and alcohol use outcome models were then conducted with the sample stratified by pregnancy status. Logistic regression models were used to estimate unadjusted and adjusted odds ratios and $95 \%$ CIs between insurance status and past month tobacco and alcohol use in the stratified models. Data were analyzed using Stata/SE statistical software version 14 (StataCorp, 2015) and used Taylor series linearization to adjust standard errors for the effects of the weighting and clustering on the precision of estimates (Wolter, 1985). All analyses accounted for the complex survey design of the NSDUH, and the final person-level analysis weight in the NSDUH was divided by four to account for the four survey years used in the analyses following the adjustment procedures described in the NSDUH codebook (SAMHSA, 2013b). Imputation-revised or recoded variables were used in these analyses. The imputation-revised variables account for missing data through statistical imputation, and serves as the basis for many recoded variables. The NSDUH recommends using the imputationrevised or recoded variables to produced estimates when available, as opposed to raw or edited variables from the survey. Detailed information on the statistical imputation method used to create the imputation-revised variables can be found elsewhere (SAMHSA, 2013b).

\section{Results}

\subsection{Descriptive characteristics}

Descriptive characteristics among reproductive age women $(\mathrm{N}=97,788)$ are reported by pregnancy status in Table 1

a and insurance status in Table $1 \mathrm{~b}$. The majority of reproductive age women were between 26 and 44 years old (57.32\%), non-Hispanic White (57.77\%), had at least a high school education (72.12\%), were unmarried (63.67\%), and were at least $200 \%$ above the US Census Bureau's poverty thresholds (55.22\%). About half of the women used alcohol in the past month (49.18\%), fewer used tobacco (23.97\%) in the past month, and $3.28 \%$ were pregnant. The majority of reproductive age women had health insurance (81.91\%), which was similar to the total proportion of insured individuals in the U.S. population during this time (84.7\%). Among reproductive age women $11.67 \%$ were nicotine dependent in the past month, 3.51 met DSM-IV criteria for alcohol abuse in the past year, and $3.36 \%$ met DSM-IV criteria for past year alcohol dependence.

\subsection{Relationship of health insurance to alcohol and tobacco use - all reproductive age women}

In the pooled model that included both pregnant and non-pregnant women, having health insurance was associated with lower odds of past month tobacco use (AOR $=0.68 ; 95 \%$ $\mathrm{CI}=0.64-0.73)$, but higher odds of past month alcohol use ( $A O R=1.22 ; 95 \% C I=1.14-1.31$ ) (Table 2 ). Pregnancy status significantly modified the association between insurance status and past month tobacco use, and insurance status and past month alcohol use (Table 3).

\subsection{Relationship of health insurance to alcohol and tobacco use, by pregnancy status}

In the analyses stratified by pregnancy status (Table 4), among pregnant women, being insured versus uninsured was not significantly associated with past month tobacco use (AOR $=1.14$; $95 \% \mathrm{CI}=0.73-1.76)$, but was associated with lower odds of past month alcohol use $(\mathrm{AOR}=0.47 ; 95 \% \mathrm{CI}=0.27-0.82)$. Among nonpregnant women, being insured versus uninsured was associated with lower odds of past month tobacco use (AOR $=0.67 ; 95 \%$ $\mathrm{CI}=0.63,0.72$ ), and higher odds of past month alcohol use (AOR $1.23 ; 95 \% \mathrm{CI}=1.15-1.32$ ).

\subsection{Past month alcohol and tobacco use by trimester among pregnant women}

Among pregnant women there were no significant differences in the relationship between insurance status, alcohol and tobacco use in the first two trimesters. However, in the third trimester, $3.18 \%$ of insured pregnant women used alcohol in the past month 
Table 1

Descriptive characteristics by pregnancy status among US women $12-44$ years old $(\mathrm{N}=97,788)$.

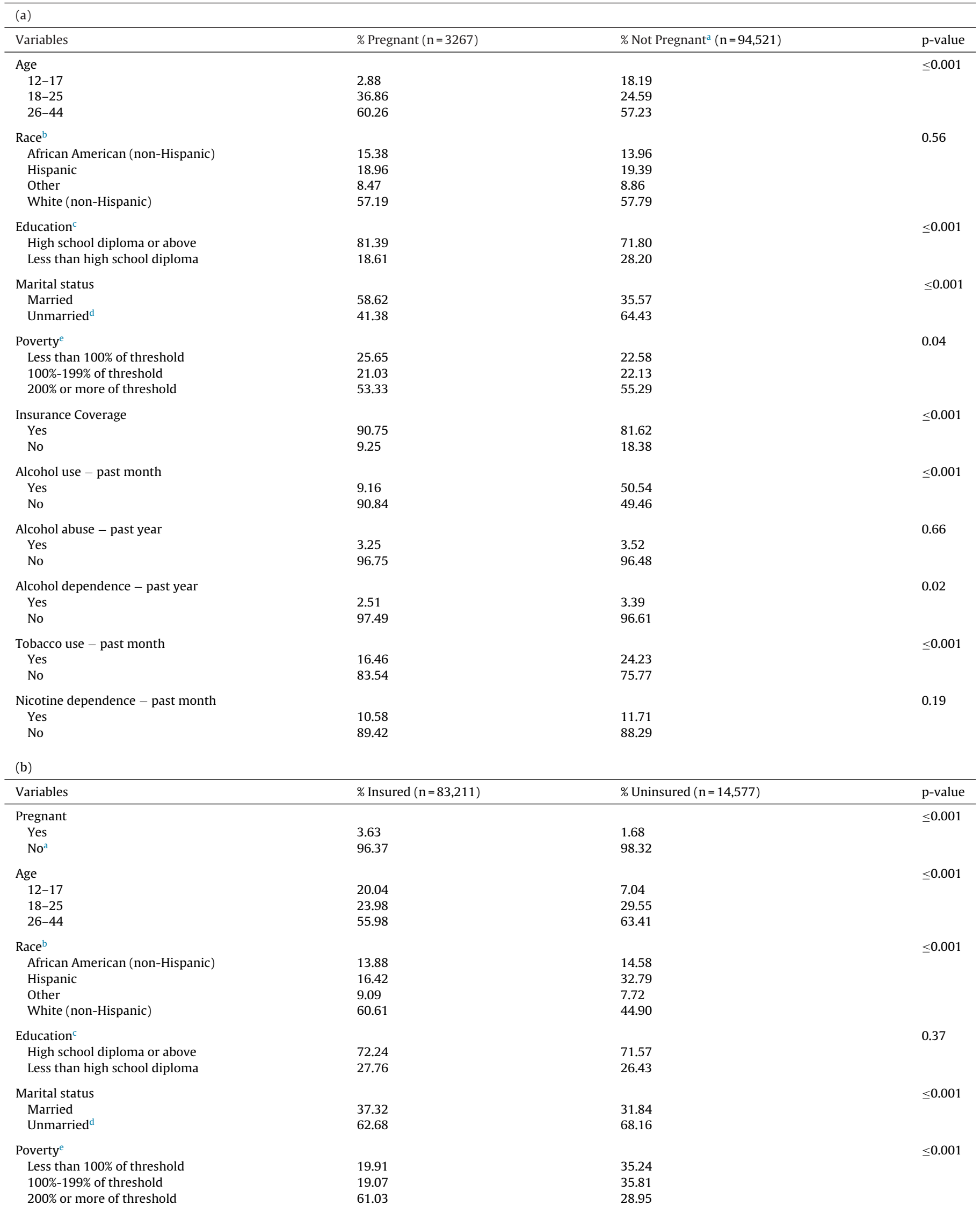


Table 1 (Continued)

\begin{tabular}{|c|c|c|c|}
\hline Variables & $\%$ Insured $(n=83,211)$ & $\%$ Uninsured $(n=14,577)$ & p-value \\
\hline Alcohol use - past month & & & $\leq 0.01$ \\
\hline Yes & 49.59 & 47.32 & \\
\hline No & 50.41 & 52.68 & \\
\hline Alcohol abuse - past year & & & 0.54 \\
\hline Yes & 3.48 & 3.68 & \\
\hline No & 96.52 & 96.32 & \\
\hline Alcohol dependence - past year & & & 0.02 \\
\hline Yes & 3.23 & 3.93 & \\
\hline No & 96.77 & 96.07 & \\
\hline Tobacco use - past month & & & $\leq 0.001$ \\
\hline Yes & 21.91 & 33.29 & \\
\hline No & 78.09 & 66.71 & \\
\hline Nicotine dependence - past month & & & $\leq 0.001$ \\
\hline Yes & 10.20 & 18.35 & \\
\hline No & 89.80 & 81.65 & \\
\hline
\end{tabular}

Data derived from the National Survey of Drug Use and Health 2010-2013.

Proportions weighted to reflect the US population.

p-values obtained from designed-based F-tests.

a Women 12-44 years old who were unsure of their pregnancy status $(\mathrm{n}=466)$ were grouped in the not pregnant reference group.

b Other race category includes non-Hispanic Native American/Alaskan Natives, non-Hispanic Hawaiians/other Pacific Islanders, non-Hispanic Asians, and people reporting more than one race (other than Hispanic).

c Persons 12-17 years old $(\mathrm{n}=35,662)$ were included in the less than high-school group.

d Participants 14 years old and younger $(n=17,211)$ were not asked questions regarding marital status (i.e., legitimate skip respondents in the NSDUH Survey). These participants were included in the unmarried group.

e Persons $18-22$ years old living in a college dorm $(n=1493)$ were grouped with persons living at $<100$ of the US Census Bureau Poverty Threshold.

Table 2

Insurance status, and past month tobacco and alcohol use among US women $12-44$ years old $(\mathrm{N}=97,788)$.

\begin{tabular}{|c|c|c|c|c|}
\hline & \multicolumn{2}{|c|}{ Odds Ratios (95\% CI) Past Month Tobacco Use } & \multicolumn{2}{|c|}{ Odds Ratios (95\% CI) Past Month Alcohol Use } \\
\hline & Unadjusted & Adjusted & Unadjusted & Adjusted \\
\hline \multicolumn{5}{|c|}{ Health Insurance } \\
\hline Yes & $0.56(0.53,0.60)^{* * *}$ & $0.68(0.64,0.73)^{* * *}$ & $1.09(1.03,1.17)^{* *}$ & $1.22(1.14,1.31)^{* * *}$ \\
\hline No & \multicolumn{2}{|l|}{ (1) } & \multicolumn{2}{|l|}{ 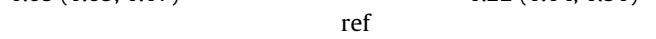 } \\
\hline
\end{tabular}

Data derived from the National Survey of Drug Use and Health 2010-2013.

Estimates weighted to reflect the US population.

Unadjusted and adjusted logistic regression models and 95\% confidence intervals.

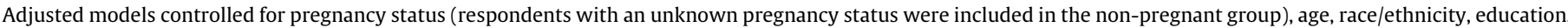

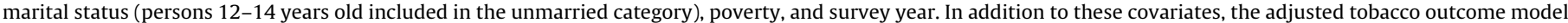
controlled for past month alcohol use, and the adjusted alcohol outcome model controlled for past month tobacco use.

**** $\mathrm{p} \leq 0.001$.

${ }^{* *} \mathrm{p} \leq 0.01$.

Table 3

Interactions between pregnancy status and insurance status among US pregnant and non-pregnant women $12-44$ years old $(\mathrm{N}=97,788)$

\begin{tabular}{lll}
\hline Past Month Alcohol Use & Past Month Tobacco Use & Adjusted Odds Ratio (95\% CI) \\
\cline { 2 - 3 } & Adjusted Odds Ratio (95\% CI)
\end{tabular}

Interaction

Health Insurance X Pregnancy Status

$1.69(1.15,2.48)^{*}$

$0.41(0.23,0.72)^{* *}$

Data derived from the National Survey of Drug Use and Health 2010-2013.

Estimates weighted to reflect the US population.

Adjusted logistic regression models with 95\% confidence intervals (CIs).

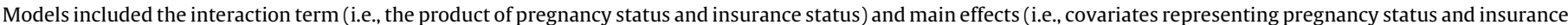

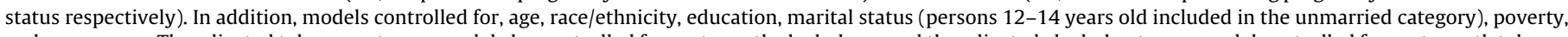

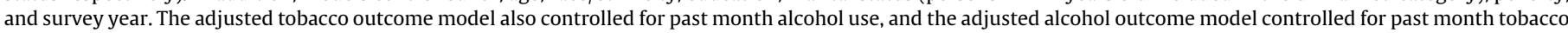
use in addition to the aforementioned covariates.

*** $\mathrm{p} \leq 0.01$. 
Table 4

The relationship between insurance status and past month tobacco and alcohol use stratified by pregnancy status among US women $12-44$ years old ( $\mathrm{n}=97,788$ ).

\begin{tabular}{|c|c|c|c|c|c|c|c|c|}
\hline & \multicolumn{4}{|c|}{ Tobacco Use - past month } & \multicolumn{4}{|c|}{ Alcohol Use - past month } \\
\hline & \multicolumn{2}{|c|}{ Pregnant women $n=3267$} & \multicolumn{2}{|c|}{ Non-pregnant women $n=94,521$} & \multicolumn{2}{|c|}{ Pregnant women n= 3267} & \multicolumn{2}{|c|}{ Non-pregnant women $n=94,521$} \\
\hline & Unadjusted & Adjusted & Unadjusted & Adjusted & Unadjusted & Adjusted & Unadjusted & Adjusted \\
\hline \multicolumn{9}{|c|}{ Health Insurance } \\
\hline $\begin{array}{l}\text { Yes } \\
\text { No }\end{array}$ & $\begin{array}{l}0.88(0.60,1.29) \\
\text { ref }\end{array}$ & $\begin{array}{l}1.14(0.73,1.76) \\
\text { ref }\end{array}$ & $\begin{array}{l}0.56(0.53,0.60)^{* * *} \\
\text { ref }\end{array}$ & $\begin{array}{l}0.67(0.63,0.72)^{* * * *} \\
\text { ref }\end{array}$ & $\begin{array}{l}0.60(0.33,1.09) \\
\text { ref }\end{array}$ & $\begin{array}{l}0.47(0.27,0.82)^{* *} \\
\text { ref }\end{array}$ & $\begin{array}{l}1.14(1.07,1.21)^{* * *} \\
\text { ref }\end{array}$ & $\begin{array}{l}1.23(1.15,1.32)^{* * *} \\
\text { ref }\end{array}$ \\
\hline
\end{tabular}

Data derived from the National Survey of Drug Use and Health 2010-2013.

Estimates weighted to reflect the US population.

Logistic regression models - estimates are odds ratios with 95\% confidence intervals (CIs)

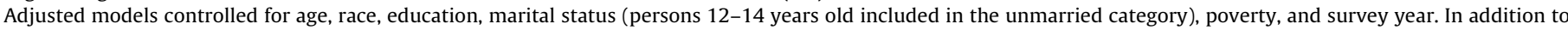

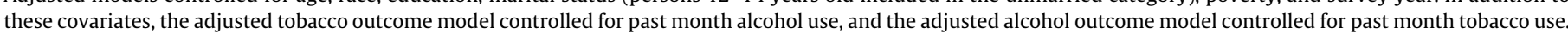
Respondents with an unknown pregnancy status were included in the non-pregnant group $(n=466)$.

**** $\mathrm{p} \leq 0.001$.

$\mathrm{p} \leq 0.01$.

Table 5

Percent of pregnant women who used alcohol and tobacco in the past month by trimester and insurance status $(\mathrm{N}=3267)$.

\begin{tabular}{|c|c|c|c|c|c|c|c|c|c|c|c|c|}
\hline & \multicolumn{4}{|c|}{ 1st Trimester $(n=1022)$} & \multicolumn{4}{|c|}{ 2nd Trimester $(\mathrm{n}=1174)$} & \multicolumn{4}{|c|}{ 3rd Trimester $(n=1071)$} \\
\hline & Insured & Uninsured & Total $^{\mathrm{a}}$ & p-value & Insured & Uninsured & Total $^{\mathrm{a}}$ & p-value & Insured & Uninsured & Total $^{\mathrm{a}}$ & p-value ${ }^{*}$ \\
\hline Alcohol & 17.91 & 24.30 & 18.81 & 0.28 & 6.05 & 5.32 & 5.99 & 0.79 & 3.18 & 0.15 & 3.02 & $\leq \mathbf{0 . 0 0 1}$ \\
\hline Tobacco & 21.45 & 23.81 & 21.79 & 0.60 & 14.42 & 13.39 & 14.34 & 0.85 & 13.60 & 11.21 & 13.48 & 0.59 \\
\hline
\end{tabular}

Estimates weighted to reflect the US population.

All models accounted for the complex survey design of the NSDUH.

* p-values obtained from designed-based F-tests; bolded values are statically significant.

a Totals are the percent of pregnant women who used alcohol or tobacco per each trimester.

versus $0.15 \%$ of uninsured pregnant women $(\mathrm{p} \leq 0.001)$ (Table 5$)$. The relationship between insurance status and tobacco use was not significant in the third trimester.

\section{Discussion}

\subsection{Summary and implications}

This study examined the relationship between health insurance coverage and past month tobacco and alcohol use among a nationally representative sample of reproductive age women in the United States, and further examined whether pregnancy status moderated these relationships. Three central findings emerged from this study. First, pregnancy status significantly moderated the relationship between health insurance status and past month tobacco and alcohol use, such that having health insurance was associated with lower odds of past month tobacco use among nonpregnant women, but importantly, not among pregnant women. Second, health insurance was associated with greater odds of alcohol use among non-pregnant women. Third, health insurance was associated with lower odds of past month alcohol use among pregnant women.

We had hypothesized that pregnancy status would moderate the relationship between health insurance and tobacco and alcohol use, such that being covered by any health insurance would be associated with lower odds of tobacco and alcohol use among both pregnant and non-pregnant women, but the magnitude of association would be stronger among pregnant women. Pregnancy status did prove to be a significant moderator, and having health insurance was significantly associated with lower odds of tobacco use among non-pregnant women, and lower odds of alcohol use among pregnant women. However, contrary to the hypotheses, health insurance coverage was not significantly associated with tobacco use among pregnant women, and was associated with increased odds of alcohol use among non-pregnant women of reproductive age.
A large literature supports health benefits, such as lower risk of mortality and cardiovascular disease, associated with low to moderate levels of alcohol use (Gunzerath et al., 2004; O'Keefe et al., 2007). The positive association between such drinking and health may be conveyed to non-pregnant women during medical visits, which would be more likely among those with insurance. However, even if not conveyed during medical visits - given that only about 1 in 6 non-pregnant women report ever having a conversation with their health care provider about alcohol use (McKnight-Eily et al., 2014) - such knowledge on the part of medical providers would preclude providing drinking-reduction advice to women who appear to have little need for such advice. Such women could engage in low to moderate drinking as part of a healthy lifestyle, which may help explain the positive relationship between any past month alcohol use and health insurance among the non-pregnant women in this study.

In contrast, the risk for fetal alcohol spectrum disorder (Jonsson et al., 2014) has been widely publicized in the United States since the 1970s (Warren, 2015). This has included a Surgeon General advisory to avoid alcohol entirely during pregnancy, and FDA and Congressional efforts leading to the Alcoholic Beverage Labeling Law that includes warnings not to drink during pregnancy. Thus, the risks of drinking alcohol during pregnancy are likely to be widely known to the medical profession, and conveyed during medical visits. Such preventive counseling has been associated with reduced odds of alcohol use during pregnancy (Elsinga et al., 2008). This would lead to lower risk for drinking among pregnant women with health insurance, as women with insurance are more likely to have health counseling to prepare for healthy pregnancy, have more prenatal visits, and present for prenatal care earlier than uninsured women (D'Angelo et al., 2007; Egerter et al., 2002; Jarvis et al., 2011), which increases their exposure to health messages and screening (Jarvis et al., 2011). This helps explain the lower odds of alcohol use among pregnant women in this study who were insured versus uninsured. Furthermore, nearly a quarter of uninsured pregnant women in their first trimester $(24.3 \%)$ used alcohol in the past month (Table 5). This suggests missed opportunities for early 
prevention of prenatal alcohol use among pregnant women who are uninsured. While in the third trimester, a significantly higher proportion of insured versus uninsured pregnant women used alcohol in the past month (3.18\% versus $0.15 \%$, respectively), the lower odds of alcohol use among those with insurance in the fully adjusted model were largely driven by the much larger total proportion of pregnant women who drank during the first trimester (18.81\%), compared to the second (5.99\%) and third (3.02\%) trimesters. Prenatal visits are optimal opportunities to begin tailoring interventions to prevent alcohol use among pregnant women, however some women report never being asked by medical providers about alcohol use during pregnancy, or counseled on the harms of use while pregnant (Cheng et al., 2011; McKnight-Eily et al., 2014). Thus, continued efforts are needed to encourage medical providers to engage women in discussion and advice or counseling about the potential harms of drinking during pregnancy.

While the lower odds for tobacco use among non-pregnant women with health insurance was expected, the lack of a significant relationship between health insurance and tobacco use among the pregnant women was not. There is no safe level of exposure to tobacco smoke for anyone (US Department of Health and Human Services, 2010), and the health consequences of tobacco use to both mother and baby can be serious (Dietz et al., 2010; Holtrop et al., 2010; Mund et al., 2013; O’Brien and Hill, 2014). Our finding in pregnant women calls attention to the need for greater health provider attention to smoking among pregnant women. According to our results (Table 5), nearly $22 \%$ of pregnant women used tobacco within the past month in their first trimester, and nearly $14 \%$ used in their third trimester. This suggest missed opportunities for tobacco use prevention at prenatal visits and also helps explain the null relationship between health insurance and tobacco use among the pregnant women. Few obstetricians follow up with their pregnant patients about smoking status after the initial prenatal visit, even if patients have acknowledged tobacco use (Coleman-Cowger et al., 2014). Health providers state that lack of time is the primary barrier to tobacco cessation interventions during prenatal visits (ColemanCowger et al., 2014). Systems of care should be modified to make more time available for physicians and other health care providers to engage pregnant women in tobacco cessation efforts.

Tobacco (Holtrop et al., 2010; Mund et al., 2013) and alcohol use (Hankin, 2002; Li et al., 2012; Maier and West, 2001; Terplan et al., 2014) are preventable risk factors of poor health for mother and baby. However, for these major risk factors to be modified, a systems-level approach should be implemented that creates a prevention framework for effectively helping to reduce tobacco and alcohol use during pregnancy. This includes research to examine factors promoting practitioner engagement of pregnant women who smoke and drink. For example, using the evidencebased " 5 As" of tobacco cessation recommended by the American College of Obstetricians and Gynecologists (providers ask about

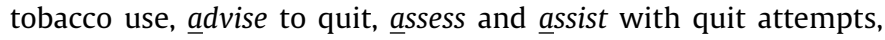
and arrange for follow-up (American College of Obstetricians and Gynecologists ACOG, 2010; Coleman-Cowger et al., 2014) is one way to help prevent prenatal tobacco use. In one state-wide survey of obstetrician/gynecologists' use of the 5 As, nearly all providers asked their patients about tobacco use, but fewer engaged in advising, assessing and assisting patients with quit attempts, and only $6 \%$ of providers arranged for follow-up visits regarding quitting tobacco use among their pregnant patients who smoked (Jordan et al., 2006), calling attention to the importance of practitioner engagement. Regarding alcohol use, and the severity of health effects associated with drinking during pregnancy (e.g., fetal alcohol spectrum disorders), the Centers for Disease Control and Prevention (CDC) recommends that women who are pregnant or might become pregnant abstain from drinking completely (CDC, 2016). In addition, the CDC recommends that providers conduct alcohol screening and brief counseling for all women; recommend birth control, when appropriate, to sexually active women who are not planning to get pregnant but who drink alcohol; advise women to stop drinking if they are trying to become pregnant; refer women to additional preventive services if they cannot stop drinking on their own; and to follow-up on drinking status of reproductive age women at least yearly, or more often if needed (CDC, 2016). Additionally, given that tobacco and alcohol use during pregnancy are often comorbid with mental health and substance use disorders (Chisolm et al., 2009a,b; Goodwin et al., 2007; Kotrla, 2008), practitioners may often focus on these other conditions in pregnant women, neglecting cigarette smoking and the importance of reducing this during pregnancy. Socioenvironmental determinates of health should also be examined, because factors such as transportation barriers or competing priorities may prevent women from engaging in necessary treatment and prevention efforts.

\subsection{Limitations and strengths}

Limitations of the study are noted. First, the cross-sectional study design does not allow for assumptions about the temporal order of the variables, which limits causal inferences. Moreover, alcohol and tobacco use was self-reported, so there was a possibility for both recall bias and social desirability bias. However, such biases were reduced by the confidential, self-administered nature of the questionnaire. Additionally, health insurance is but one of many ways to operationalize access to health care. Future studies should explore other indicators of access to health care and associated correlates to provide a more complete picture of factors that influence tobacco and alcohol use among reproductive age women. Furthermore, nicotine dependence and alcohol use disorders were not examined in the primary analyses, but this is potentially an informative direction to go in future analyses of the topic of insurance status and alcohol and tobacco use among reproductive age women.

This study also has several important strengths. Over three thousand pregnant women were available from the surveys and included in the analyses. The data are nationally representative, which lends to the generalizability of the results. Additionally, this study highlights the pervasiveness of tobacco use during pregnancy and calls for future research to examine mechanisms that explain and may potentially reduce tobacco use among pregnant women.

\section{Conclusions}

Providing access to health care via health insurance coverage appears to be important in helping reduce the risk of alcohol use during pregnancy. However, despite health insurance coverage, tobacco use persists during pregnancy, potentially suggesting missed opportunities for prevention during prenatal visits.

\section{Role of funding source}

This work was supported by research grants from the National Institute on Drug Abuse (grant number T32DA031099 (P.I. Hasin); 1R01DA037866 (P.I. Martins), the National Institute on Alcohol Abuse and AlcoholismK01AA021511 (P.I. Keyes) and the New York State Psychiatric Institute (Hasin). The sponsoring grant agencies had no further role in the study design and analysis, the writing of the report, or the decision to submit the paper for publication. The Substance Abuse and Mental Health Data Archive provided the public use data files for NSDUH, which was sponsored by the Office of Applied Studies of the Substance Abuse and Mental Health Service Administration. 


\section{Contributors}

All authors made substantial contributions to the paper: Brown and Martins developed the concept and study design. Brown conducted the statistical analyses. Martins, Hasin, Keyes, and Fink provided feedback on the analyses. Brown and Hasin drafted the manuscript. Brown, Martins, Hasin, Keyes, Fink, and Ravenell contributed to the interpretation of the findings. All authors provided critical revisions to the manuscript and approved the final version.

\section{Conflict of interest}

\section{No conflicts declared.}

\section{References}

Adams, E.K., Markowitz, S., Dietz, P.M., Tong, V.T., 2013. Expansion of Medicaid covered smoking cessation services: maternal smoking and birth outcomes. Medicare Medicaid Res. Rev. 3.

American College of Obstetricians Gynecologists ACOG, 2010. Committee opinion no. 471: smoking cessation during pregnancy. Obstet. Gynecol. 116, 1241-1244.

Beronio, K., Glied, S., Po, R., Skopec, L., 2013. Affordable Care Act Will Expand Mental Health And Substance Use Disorder Benefits And Parity Protections For 62 Million Americans. Department of Health and Human Services, Washington, DC

Biondo, G., Chilcoat, H.D., 2014. Discrepancies in prevalence estimates in two national surveys for nonmedical use of a specific opioid product versus any prescription pain reliever. Drug Alcohol Depend. 134, 396-400.

Centers for Disease Control and Prevention, 2016. Vital Signs: Alcohol And Pregnancy Why Take The Risk?, http://www.cdc.gov/vitalsigns/fasd/. (accessed 09.04.16).

Chen, J., Vargas-Bustamante, A., Mortensen, K., Ortega, A.N., 2015. Racial and ethnic disparities in health care access and utilization under the affordable care act. Med. Care 54, 140-146.

Cheng, D., Kettinger, L., Uduhiri, K., Hurt, L., 2011. Alcohol consumption during pregnancy: prevalence and provider assessment. Obstet. Gynecol. 117, 212-217.

Chisolm, M.S., Tuten, M., Brigham, E.C., Strain, E.C., Jones, H.E., 2009a. Relationship between cigarette use and mood/anxiety disorders among pregnant methadone-maintained patients. Am. J. Addict. 18, 422-429.

Chisolm, M.S., Tuten, M., Strain, E.C., Jones, H.E., 2009b. Screening for mood disorder in pregnant substance-dependent patients. Addict. Disord. Their Treat. 8, 88-98 (11p).

Coleman-Cowger, V.H., Anderson, B.L., Mahoney, J., Schulkin, J., 2014. Smoking cessation during pregnancy and postpartum: practice patterns among obstetrician-gynecologists. J. Addict. Med. 8, 14-24.

D’Angelo, D., Williams, L., Harrison, L., Ahluwalia, I., 2012. Health status and health insurance coverage of women with live-born infants: an opportunity for preventive services after pregnancy. Maternal Child Health J. 16, 222-230.

D’Angelo, D., Williams, L., Morrow, B., Cox, S., Harris, N., Harrison, L., Posner, S.F., Hood, J.R., Zapata, L., 2007. Preconception and interconception health status of women who recently gave birth to a live-born infant - Pregnancy Risk Assessment Monitoring System (PRAMS), United States, 26 reporting areas, 2004. MMWR 56, 1-35.

D’Angelo, D.V., Le, B., O'Neil, M.E., Williams, L., Ahluwalia, I.B., Harrison, L.L., Floyd, R.L., Grigorescu, V., 2015. Patterns of health insurance coverage around the time of pregnancy among women with live-born infants-pregnancy risk assessment monitoring system, 29 states, 2009. MMWR Surveill. Summ. 64, $1-19$.

Department of the Treasury, Department of Labor, Department of Health and Human Services, 2013. Final rules under the Paul Wellstone and Pete Domenici Mental Health Parity and Addiction Equity Act of 2008; technical amendment to external review for multi-state plan program. Final rules. In: Department of the Treasury, D.o.L., Department of Health and Human Services (Ed.). Office of the Federal Register, National Archives and Records Service, General Services Administration; distribution by the Supt. of Docs., U.S. Govt. Print. Off. [etc.] United States. pp. 68239-68296.

Dietz, P.M., England, L.J., Shapiro-Mendoza, C.K., Tong, V.T., Farr, S.L., Callaghan, W.M., 2010. Infant morbidity and mortality attributable to prenatal smoking in the U.S. Am. J. Prev. Med. 39, 45-52.

Egerter, S., Braveman, P., Marchi, K., 2002. Timing of insurance coverage and use of prenatal care among low-income women. Am. J. Public Health 92, 423-427.

Elsinga, J., de Jong-Potjer, L.C., van der Pal-de Bruin, K.M., le Cessie, S., Assendelft, W.J., Buitendijk, S.E., 2008. The effect of preconception counselling on lifestyle and other behaviour before and during pregnancy. Womens Health 18, S117-125.

Ethen, M.K., Ramadhani, T.A., Scheuerle, A.E., Canfield, M.A., Wyszynski, D.F. Druschel, C.M., Romitti, P.A., 2009. National Birth Defects Prevention Study, 2009. Alcohol consumption by women before and during pregnancy. Maternal Child Health J. 13, 274-285.
Floyd, R.L., Jack, B.W., Cefalo, R., Atrash, H., Mahoney, J., Herron, A., Husten, C. Sokol, R.J., 2008. The clinical content of preconception care: alcohol, tobacco, and illicit drug exposures. Am. J. Obstet. Gynecol. 199, S333-339.

Goodwin, R.D., Keyes, K., Simuro, N., 2007. Mental disorders and nicotine dependence among pregnant women in the United States. Obstet. Gynecol. 109, 875-883.

Gunzerath, L., Faden, V., Zakhari, S., Warren, K., 2004. National Institute on Alcohol Abuse and Alcoholism report on moderate drinking. Alcohol. Clin. Exp. Res. 28, $829-847$

Hankin, J.R., 2002. Fetal alcohol syndrome prevention research. Alcohol. Res. Health 26, 58.

Havens, J.R., Simmons, L.A., Shannon, L.M., Hansen, W.F., 2009. Factors associated with substance use during pregnancy: results from a national sample. Drug Alcohol Depend. 99, 89-95.

Holtrop, J.S., Meghea, C., Raffo, J.E., Biery, L., Chartkoff, S.B., Roman, L., 2010. Smoking among pregnant women with medicaid insurance: are mental health factors related? Maternal Child Health J. 14, 971-977.

Jarlenski, M., Bleich, S.N., Bennett, W.L., Stuart, E.A., Barry, C.L., 2014. Medicaid enrollment policy increased smoking cessation among pregnant women but had no impact on birth outcomes. Health Affairs 33, 997-1005.

Jarvis, C., Munoz, M., Graves, L., Stephenson, R., D’Souza, V., Jimenez, V., 2011. Retrospective review of prenatal care and perinatal outcomes in a group of uninsured pregnant women. J. Obstet. Gynaecol. Can. 33, 235-243.

Jonsson, E., Salmon, A., Warren, K.R., 2014. The international charter on prevention of fetal alcohol spectrum disorder. Lancet Global Health 2, e135-e137.

Jordan, T.R., Dake, J.R., Price, J.H., 2006. Best practices for smoking cessation in pregnancy: do obstetrician/gynecologists use them in practice? J. Womens Health 15, 400-441.

Kotrla, K., 2008. Predicting alcohol use during pregnancy: analysis of national survey data and implications for practice and the church. Social Work Christianity 35, 12-32.

Kratz, L.M., Vaughan, E.L., 2012. Mental health problems, legal involvement, and smoking during pregnancy. Subst. Use Misuse 47, 718-725.

Li, Q., Hankin, J., Wilsnack, S.C., Abel, E.L., Kirby, R.S., Keith, L.G., Obican, S.G., 2012. Detection of alcohol use in the second trimester among low-income pregnant women in the prenatal care settings in Jefferson County, Alabama. Alcohol. Clin. Exp. Res. 36, 1449-1455.

Ma, Y., Goins, K.V., Pbert, L., Ockene, J.K., 2005. Predictors of smoking cessation in pregnancy and maintenance postpartum in low-income women. Maternal Child Health J. 9, 393-402.

Maier, S.E., West, J.R., 2001. Drinking patterns and alcohol-related birth defects Alcohol. Res. Health 25, 168

McKnight-Eily, L.R., Liu, Y., Brewer, R.D., Kanny, D., Lu, H., Denny, C.H., Balluz, L., Collins, J., 2014. Vital signs: communication between health professionals and their patients about alcohol use-44 states and the District of Columbia, 2011. MMWR 63, 16-22.

Muhuri, P.K., Gfroerer, J.C., 2009. Substance use among women: associations with pregnancy, parenting, and race/ethnicity. Maternal Child Health J. 13, 376-385

Mund, M., Louwen, F., Klingelhoefer, D., Gerber, A., 2013. Smoking and pregnancy-a review on the first major environmental risk factor of the unborn. Int. J. Environ. Res. Public Health 10, 6485-6499.

Newacheck, P.W., Stoddard, J.J., Hughes, D.C., Pearl, M., 1998. Health insurance and access to primary care for children. N. Engl. J. Med. 338, 513-519 (517p)

O'Brien, J.W., Hill, S.Y., 2014. Effects of prenatal alcohol and cigarette exposure on offspring substance use in multiplex: alcohol-dependent families. Alcohol. Clin. Exp. Res. 38, 2952-2961.

O'Keefe, J.H., Bybee, K.A., Lavie, C.J., 2007. Alcohol and cardiovascular health: the razor-sharp double-edged sword. J. Am. Coll. Cardiol. 50, 1009-1014 (1006).

Office of Legislative Counsel, 2010. Compilation of Patient Protection and Affordable Care Act. U.S. House of Representatives, Wahsington, DC

SAMHSA, 2010. 2010 National Survey on Drug Use and Health: Methodological Resource Book. Substance Abuse and Mental Health Services Administration, Rockville, MD.

SAMHSA, 2011. 2011 National Survey on Drug Use and Health: Methodological Resource Book. Substance Abuse and Mental Health Services Administration, Rockville, MD

SAMHSA, 2012. 2012 National Survey on Drug Use and Health: Methodological Resource Book. Substance Abuse and Mental Health Services Administration, Rockville, MD.

SAMHSA, 2013a. Center for behavioral health statistics and quality. (2014). In: 2013 National Survey on Drug Use and Health: Methodological Resource Book (Section 8, Data Collection Final Report). Substance Abuse and Mental Health Services Administration, Rockville, MD.

SAMHSA, 2013b. Center for behavioral health statistics and quality. (2014). In: 2013 National Survey on Drug Use and Health: Public Use File Codebook. Substance Abuse and Mental Health Services Administration, Rockville, MD.

StataCorp, 2015. Stata Statistical Software: Release 14. StataCorp LP, College Station, TX.

Substance Abuse and Mental Health Services Administration SAMHSA, 2012. National Survey on Drug Use and Health, 2011: Codebook. Rockville, MD.

Terplan, M., McNamara, E.J., Chisolm, M.S., 2012. Pregnant and non-pregnant women with substance use disorders: the gap between treatment need and receipt. J. Addict. Dis. 31, 342-349.

Terplan, M., Cheng, D., Chisolm, M.S., 2014. The relationship between pregnancy intention and alcohol use behavior: an analysis of PRAMS data. J. Subst. Abuse Treat. 46, 506-510. 
Tong, V.T., Jones, J.R., Dietz, P.M., D’Angelo, D., Bombard, J.M., 2009. Trends in smoking before, during, and after pregnancy - Pregnancy Risk Assessment Monitoring System (PRAMS) United States, 31 sites, 2000-2005. MMWR Surveill. Summ. 58, 1-30.

Department of Health U.S. Department of Health and Human Services, How Tobacco Smoke Causes Disease: The Biology And Behavioral Basis For Smoking-Attributable Disease: A Report Of The Surgeon General U.S.

Department Of Health And Human Services, Centers For Disease Control And Prevention, National Center For Chronic Disease Prevention and Health Promotion, Office on Smoking and Health Atlanta, GA 2010.
Warren, K.R., 2015. A review of the history of attitudes toward drinking in pregnancy. Alcohol. Clin. Exp. Res. 39, 1110-1117.

Wolter, K.M., 1985. Introduction to Variance Estimation. Springer, New York, NY.

Zuvekas, S.H., Taliaferro, G.S., 2003. Pathways to access: health insurance, the health care delivery system, and racial/ethnic disparities, 1996-1999. Health Affairs 22, 139-153. 